\title{
Los profesionales de urgencias y emergencias: perfil actual y posibilidades de desarrollo
}

\section{Urgencies and emergencies professionals: current profile and potential development}

\author{
T. Toranzo' , F. Aramburu²
}

\section{RESUMEN}

Los servicios de urgencias, tanto hospitalarios como extrahospitalarios, son dispositivos asistenciales que se han desarrollado, sobre todo, a raíz de la publicación de la Ley General de Sanidad en 1986. Tienen una alta frecuentación y gran sobrecarga asistencial, a la vez que dispensan una elevada calidad asistencial y son elementos estratégicos en el funcionamiento del Sistema Nacional de Salud. Sin embargo, sus profesionales, elementos básicos y sustanciales en su funcionamiento han estado relegados en cuanto a la planificación estratégica y, sobre todo, sin una formación especializada reglada en Medicina de Urgencias y Emergencias que garantice que, desde el mismo momento en que asumen responsabilidades asistenciales, tienen todas las competencias, habilidades y actitudes que las necesidades de los enfermos les demandan. Esta carencia también impide un correcto desarrollo profesional y es la base de la viabilidad futura de los propios servicios de urgencias. Para solventar este dilema, además de buscar soluciones a los problemas laborales y organizativos de los distintos servicios, la creación de la Especialidad de Medicina de Urgencias y Emergencias, en el marco de la troncalidad, es un requisito imprescindible. En ese sentido lo están abordando los países de nuestro entorno y así lo han reconocido, desde el Parlamento Español, hasta la mayoría de los sectores políticos, sociales y profesionales.

Palabras clave. Medicina de urgencias. Formación especializada. Desarrollo profesional.

\begin{abstract}
Emergency services, both hospital based and pre-hospital, are care mechanisms that have been developed above all as a result of the General Health Law of 1986. They are in high demand and have a care overload, while they provide high quality care and are strategic elements in the working of the National Health System. However, their professionals, basic and substantial elements in their tasks, have been relegated in strategic planning and, above all, do not receive regulated specialised training in Emergency Medicine. This would guarantee that from the very moment they take up their care duties they have all of the abilities, skills and attitudes demanded of them by the patients' needs. This lack also prevents correct professional development, which is the basis of the future viability of the emergency services themselves. To resolve this dilemma, besides seeking solutions to the work and organisational problems of the different services, the creation of the Speciality of Accident and Emergency Medicine, in the framework of core medical studies, is an essential requisite. This is how it is being dealt with by our nearby countries and this need has been recognised by the Spanish Parliament and by the majority of political, social and professional sectors.
\end{abstract}

Key words. Accident and emergency medicine. Specialised training. Professional development.
1. Presidente de SEMES. Médico de Urgencia Hospitalaria. Hospital Virgen de la Concha. Zamora.

2. Vicepresidente $1^{\circ}$ de SEMES. Médico de Urgencia Hospitalaria. Complejo Hospitalario Ourense.

\section{Correspondencia}

Tomás Toranzo Cepeda

Avda. Aldebarán, 35

Urbanización Aldebarán

37900 Santa Marta de Tormes. Salamanca

E-mail: presidente@semes.org 


\section{INTRODUCCIÓN}

Desde que en abril de 1986 se promulgó Ley General de Sanidad (Ley 14/1986) y se estableció como derecho la asistencia sanitaria pública para toda la población -complementándose posteriormente con nuevas disposiciones legales que la han hecho prácticamente gratuita y con una amplísima cartera de servicios-, se inició, unido a importantes cambios socio-culturales, demográficos y epidemiológicos, una escalada en la demanda asistencial a la que no fueron ajenos los por entonces precarios servicios o dispositivos de asistencia urgente. Así, en el último cuarto del siglo pasado, bajo una presión asistencial creciente, denuncias de profesionales, pacientes e incluso del Defensor del Pueblo en 1988 quien hizo público su "Informe sobre Servicios de Urgencia del Sistema Sanitario Público" en el que demandaba más servicios de urgencias, mejor dotados y más cualificados, se fueron creando, configurando y mejorando en España los servicios de urgencias, tanto hospitalarios como extrahospitalarios. En este momento son ya unas estructuras asistenciales consolidadas, mejor organizadas, con unas dotaciones tecnológicas que, con carácter general, son más adecuadas a las necesidades y funciones de estos servicios ${ }^{1}$.

Sin embargo, ya desde sus inicios tuvieron una carencia básica aún no solucionada hoy en día: la falta de una especialización en Medicina de Urgencias y Emergencias (MUE) que garantice que cualquier profesional que se incorpora a estos servicios tiene, desde el mismo momento de su contratación, todas las competencias, habilidades y actitudes que ese puesto de trabajo profesional requiere, en el mejor interés de los pacientes. Este problema, hasta ahora, se ha solventado con la entrega y dedicación de sus profesionales, completando cada uno sus carencias, en función de la especialidad de procedencia, con autoformación. La Sociedad Española de Medicina de Urgencias y Emergencias (SEMES), a través de su cuerpo doctrinal $^{2}$, ha tratado de orientar esa formación específica en medicina de urgencias $y$, junto con la evaluación de la experien- cia profesional y de la actividad docente e investigadora, ha servido para diseñar un currículo profesional que es la base del Certificado de Medicina de Urgencias y Emergencias (CME) de la SEMES. Su objetivo es certificar que los conocimientos y preparación de quien lo posee son los adecuados para el ejercicio profesional de la MUE en tanto en cuanto ésta no sea reconocida oficialmente como especialidad.

Esa falta de formación especializada específica en MUE ha hecho que el perfil formativo de los urgenciólogos, en sus diferentes ámbitos asistenciales, sea muy variado. Hasta hace pocos años predominaban los médicos generalistas sobre los médicos especialistas en medicina familiar y comunitaria (MFyC) y en medicina interna (MI) (Fig. 1). Pero teniendo en cuenta que, según establece el Real Decreto (RD) 853/1993, a partir del 1 de enero de 1995, para poder ejercer en el Sistema Nacional de Salud (SNS) es imprescindible estar en posesión del título de cualquier especialidad o poseer la certificación prevista en el mismo, el colectivo de generalistas ha ido decreciendo a la vez que aumentado el de especialistas en MFyC y en MI. Estos profesionales, según se van incorporando a los distintos servicios de urgencias, han tenido que completar sus carencias formativas en medicina de urgencias.

Por otro lado, la creación en el antiguo territorio Insalud de la categoría de Médicos de Urgencia Hospitalaria mediante el RD $866 / 2001$, seguido de decretos similares en otras comunidades autónomas (CC.AA.) además de regular las funciones de estos profesionales, sirvió para abrir un proceso de estabilización profesional que se generalizó en toda España, también en aquellas CC.AA. que no crearon esta categoría y también en el ámbito extrahospitalario. Es decir, la gran mayoría de los urgenciólogos que llevan más de 5 años como tales, tienen plaza en propiedad o están en proceso de conseguirla, por lo que su mayor problema laboral en estos momentos es el derivado de la sobrecarga asistencial por la insuficiencia de las plantillas, la determinación de las jornadas de trabajo y los módulos de guardia ${ }^{3}$. 


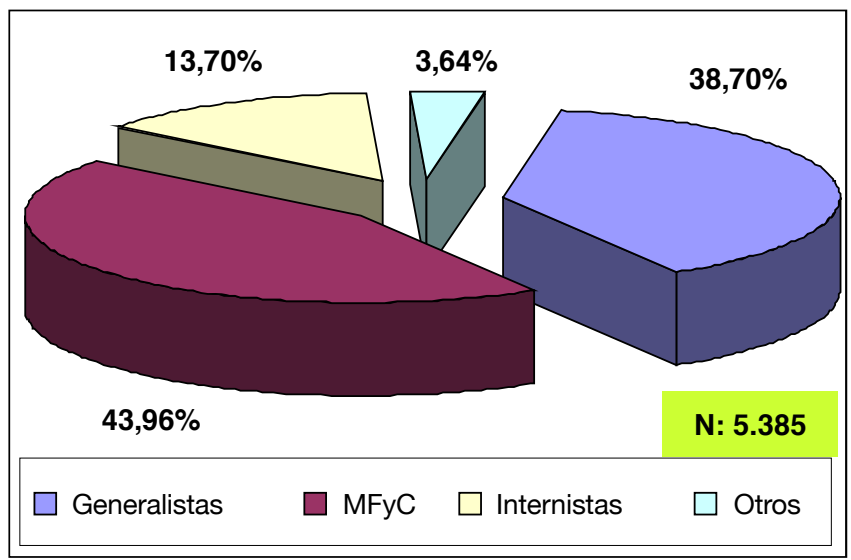

Figura 1. Titulación de los médicos de urgencia hospitalaria. Encuesta SEMES 2007.

Hoy en día los distintos servicios de urgencias disponen de plantillas con gran competencia profesional, con una calidad asistencial contrastada y con una frecuentación muy elevada que confirma que esta aseveración es percibida por la población. Así, sólo en los servicios de urgencia hospitalaria, tanto públicos como privados de toda España, en 2007, con un incremento interanual de casi un $4 \%$, se hicieron más de 26 millones de asistencias (Tabla 1$)^{4} \mathrm{y}$, aunque muchas de ellas no fuesen adecuadas a este nivel asistencial, una de las principales razones que aducen los pacientes para acudir a urgencias es la confianza que estos servicios y sus profesionales les merecen ${ }^{5}$.

Tabla 1. Urgencias atendidas en hospitales en España 2001-2007.

\begin{tabular}{|c|c|c|c|}
\hline \multirow{2}{*}{ Urgencias } & Año 2001 & Año 2007 & \% Variación \\
\hline & 21.324 .816 & 26.265 .096 & 23,16 \\
\hline
\end{tabular}

Esta alta frecuentación también es una de las causas de las enormes cargas asistenciales que se soportan existiendo una clara inadecuación entre la oferta y la demanda ${ }^{6}$, tanto en lo que se refiera a recursos materiales (camas, boxes...) como humanos, sobre todo sanitarios, y eso que los urgenciólogos ya representan el 9,7\% del total de médicos hospitalarios y la enfermería de urgencias el 8,6\% de la enfermería hospitalaria ${ }^{5}$. A todo esto hay que añadir la insuficiencia de su modelo organizativo y de gestión, fundamentalmente en lo que a su autonomía se refiere, y a los problemas de interrelación con el resto del sistema sa- nitario, sobre todo con el propio hospital. Y es que, aunque sólo el 10,5\% de las urgencias son ingresadas, los servicios de urgencia tienen una importancia estratégica en el funcionamiento hospitalario, ya que su actividad genera (Tabla 2) más de la mitad del total de sus ingresos (algo más del 62\% si sólo nos referimos a los hospitales públicos $)^{7}$. Sin embargo, a pesar de eso, con demasiada frecuenta la planificación de la asistencia hospitalaria en general (ingresos, consultas externas, etc.) se hace sin tener presente la actividad de los servicios de urgencias, lo cual cierra el círculo de la saturación de las urgencias, circunstancia 
que, además de repercutir directamente en la calidad asistencial que reciben los pacientes, es fuente de conflicto con los profesionales.

Tabla 2. Actividad en el Servicio de Urgencias de hospitales de España. Año 2007.

\begin{tabular}{ccccc}
\hline Total de urgencias & $\begin{array}{c}\text { \% de urgencias del } \\
\text { SNS }\end{array}$ & $\begin{array}{c}\text { \% de urgencias } \\
\text { ingresadas }\end{array}$ & $\begin{array}{c}\text { Frecuentación } \\
\text { por mil hab. }\end{array}$ & $\begin{array}{c}\text { \% Presión } \\
\text { Urgencias }\end{array}$ \\
26.265 .096 & $79,42 \%$ & $10,50 \%$ & 585,31 & 52,47 \\
\hline
\end{tabular}

SNS: Sistema Nacional de Salud

Hab: habitantes

Por último, uno de los elementos que mejor definen las virtudes de nuestro SNS es la equidad y ésta adquiere todo su sentido en aquellas circunstancias en que las necesidades del paciente son tiempo dependientes, en las que puede que no exista una segunda oportunidad y que se presentan en los lugares más diversos, lejos de los grandes recursos de la medicina moderna. Ahí, el profesional perfectamente formado, sin carencias competenciales y con habilidades y actitudes para solucionar cualquier necesidad del paciente, va a ser la diferencia entre la vida y la muerte.

\section{LA TRONCALIDAD EN LA FORMACIÓN ESPECIALIZADA}

Con independencia de los déficits laborales citados, la falta de expectativas profesionales (sobre todo ligadas a la carencia de especialidad y a la posibilidad de tener residentes específicos que, además de estimular la formación continuada, la docencia y la investigación, garanticen en el futuro el relevo generacional y anulen el mecanismo perverso de aceptar contratos en urgencias en espera de un contrato en la especialidad de origen) se cita con frecuencia como uno de los elementos clave que lleva a los urgenciólogos a abandonar sus actuales puestos de trabajo. Esto pone a los servicios de urgencias, hospitalarios y extrahospitalarios, en una difícil coyuntura en cuanto a su viabilidad futura, tanto desde el punto de vista de continuidad física como de la vertiginosa pérdida de calidad, que hasta entonces se iría produciendo y donde los más perjudicados serían los propios pacientes.
Así, en los últimos años, esgrimiendo estos argumentos, numerosos urgenciólogos, con larga trayectoria en estos servicios, los han abandonado ${ }^{8}$ buscando ámbitos asistenciales más cómodos y, a veces, mejor retribuidos y, sobre todo, con mejor reconocimiento social. Hasta los médicos en periodo de formación (MIR) que rotan en urgencias tiene un elevado grado de burnout $t^{9}$ y se niegan a que se les utilice como "mano de obra" en servicios para los que no se están formando. Incluso han protagonizado sonoras protestas a las puertas de los hospitales (Hospital La Fe de Valencia, septiembre de 2009). No ocurre lo mismo en otros países, donde la especialidad está perfectamente consolidada, como en EE.UU., donde las plazas de urgenciólogo tiene una demanda profesional muy elevada y un gran reconocimiento social y profesional ${ }^{10}$.

La troncalidad en la formación especializada, tal y como se prevé en la Ley 44/2003 de Ordenación de las Profesiones Sanitarias, junto con la creación por RD de la especialidad de MUE, será la oportunidad para acabar con estas amenazas. Así lo entendió el Parlamento Español, así lo han ratificado los dos últimos ministros de $\mathrm{Sa}$ nidad y así lo manifiesta un amplio consenso político, técnico ${ }^{11}$ y profesional. Existen, también, algunos e influyentes detractores. En la Unión Europea (UE) desde hace años se camina por esa senda y la European Society for Emergency Medicine (EuSEM) y la Union Européenne des Médecins Spécialistes (UEMS), con gran respaldo profesional, han propuesto un plan de estudios que homogenice esta formación en la $\mathrm{UE}^{12}$, sobre todo teniendo en cuenta que ya son 14 los 
países que tiene reconocida una formación especializada en MUE ${ }^{13,14}$. Después, con la especialidad de MUE conseguida, siguiendo los valores del moderno profesionalismo ${ }^{15-16}$, consolidaríamos un modelo asistencial que responde exitosamente a las necesidades actuales de los pacientes, da respuesta eficaz a las demandas sociales y es acorde a los valores profesionales.

A pesar de todo, aún estamos esperando y la paciencia y las oportunidades se acaban. El tiempo no se recupera. También la especialidad de MUE es tiempo dependiente. Así, parafraseando a Hipócrates, se puede decir que "La vida es corta y el arte, largo; la ocasión, fugaz; el experimento, peligroso y el juicio, difícil». Por eso, no sólo se debe estar preparado para cumplir con el deber, sino que también se debe buscar la cooperación del paciente, de los ayudantes y de las circunstancias externas. Es decir, que para conseguir la especialidad y garantizar nuestro desarrollo profesional, en el mejor interés de los pacientes, no podemos permitirnos el lujo de no hacer todo el esfuerzo posible, ni podemos despreciar ninguna colaboración. Es nuestra obligación profesional. Pero, si fracasamos en el intento, todos seríamos perdedores.

\section{BIBLIOGRAFÍA}

1. Aramburu Vilariño FJ. Los Servicios de Urgencias y la Medicina General. Siglo XXI. Emergencias 2001; 13: 4-7.

2. Comité Científico SEMES. Medicina de Emergencias. Cuerpo doctrinal. Emergencias 1996; 8: 73-80.

3. Montero Pérez FJ, Calderón de la Barca Gázquez JM, Jiménez Murillo L, Berlango Jiménez A, PÉrez Torres I, PÉrula de Torres L. Situación actual de los Servicios de Urgencias Hospitalarios en España (III): recursos materiales y humanos. Perfil profesional del médico de Urgencias. Emergencias 2000; 12: 248-258.

4. Instituto de información sanitaria. Estadística de establecimientos sanitarios con régimen de internado. Indicadores hospitalarios: evolución 2000-2005 (consultado el 3 de diciembre de 2009). Disponible en: http:// www.msc.es/estadEstudios/estadisticas/ docs/serie_escri_00_05_es.pdf
5. Ministerio de Sanidad y Política Social. Instituto de información sanitaria. Barómetro sanitario 2008 (consultado el 28 de noviembre de 2009). Disponible en: http://www.msc. es/estadEstudios/estadisticas/docs/LIBROBS2008.pdf

6. SÁNCHEz M. ¿Urgencias inadecuadas u oferta insuficiente? Editorial. Med Clin (Barc) 2004; 123: 619-620.

7. Ministerio de Sanidad y Política Social. Instituto de información sanitaria. Estadística de establecimientos sanitarios con régimen de internado. Indicadores hospitalarios año 2007 (consultado el 2 de diciembre de 2009). Disponible en: http://www.msc.es/estadEstudios/estadisticas/docs/EESCRI_2007.pdf

8. Diario Médico (consultado el 28 de noviembre de 2009). Disponible en: http://www.diariomedico.com/2008/07/10/area-profesional/ profesion/el-sns-pierde-en-9-meses-a-574urgenciologos

9. Fernández Martínez O, Hidalgo Cabrera C, Martín Tapia A, Moreno Suárez S, García del Río GARcía B. Burnout en médicos residentes que realizan guardias en un servicio de urgencias. Emergencias 2007; 19: 116-121.

10. Center for Health Workforce Studies. The Emergency Care Workforce in the U.S. August 2006 (consultado el 3 de diciembre de 2009). Disponible en: http://www.albany. edu/news/pdf_files/EmergencyCare\%20Workforce\%20in\%20the\%20US\%2008-2006A.pdf

11. Grupo de trabajo de Urgencias y Emergencias. Ministerio de Sanidad y Consumo. Perfil del Médico de Urgencias y Emergencias. Madrid, 9 de marzo de 2006.

12. Grupo de trabajo de la EuSEM (European Society for Emergency Medicine) sobre el plan de estudios aprobado por el Consejo y la Federación de las Sociedades Nacionales de la EuSEM y por el Comité Mixto UEMS (Unión Européenne des Médecins Spécialistes) multidisciplinario en medicina de emergencia. Plan Europeo de Estudios de Medicina de Urgencias y Emergencias. Emergencias 2009; 21: 456-470.

13. Diario Oficial de la Unión Europea. (ES) 30/09/2005. L 255:22-142 Directiva 2005/36/ CE del Parlamento Europeo y del Consejo, de 7 de septiembre de 2005 relativa al reconocimiento de cualificaciones profesionales.

14. Diario Oficial de la Unión Europea. (ES) 20/12/2006. 141-237 Directiva 2005/36/CE del Parlamento Europeo y del Consejo, de 20 de noviembre de 2006 por la que se adaptan determinadas directivas en el ámbito de la 
libre circulación de personas, con motivo de la adhesión de Bulgaria y Rumania.

15. Pardell $\mathrm{H}$. El nuevo profesionalismo médico. Una ideología expresada en conductas. En Oriol-Bosch A, Pardell H, eds. La profesión médica. Los retos del milenio. Barcelona: Fundación Medicina y Humanidades Médicas; 2005: 11-22.

16. WoJtczaK A. Profesionalismo médico. Una problemática global. Educ Med 2006; 9: 144-145. 